\section{Mortalidade por causas mal definidas, Brasil, 1979-2002, e um modelo preditivo para idade}

\author{
Mortality due to ill-defined causes in Brazil \\ (1979-2002) and a predictive model for age
}

\author{
${ }_{1}^{1}$ Fundação Sistema Estadual \\ de Análise de Dados, \\ São Paulo, Brasil. \\ 2 Departamento de Medicina \\ Preventiva, Universidade \\ Federal de São Paulo \\ São Paulo, Brasil. \\ Correspondência \\ M. R. Costa \\ Fundação Sistema Estadual \\ de Análise de Dados. \\ Rua Hematita 223, \\ São Paulo, SP \\ 05180-120, Brasil. \\ mcosta@seade.gov.br
}

\section{Abstract}

This study focused on the percentage of deaths due to ill-defined causes in Brazil, from 1979 to 2002. The objectives were to: (a) describe changes over the 24-year period; (b) identify the age group in which the percentage of ill-defined causes correlated most closely with the total percentage of ill-defined causes; (c) select a predictive model for the percentage of ill-defined causes in such age group, given the total percentage of ill-defined causes; (d) describe changes in the distributions of each age group in the total ill-defined causes; and (e) verify whether the percentage of deaths in-hospital shows any relationship to the percentage of ill-defined causes. Results showed that the total percentage of deaths from ill-defined causes decreased in Brazil. The percentage of ill-defined causes in the $\geq 50$-year age group correlated most closely with the total percentage of ill-defined causes, and cubic regression was the most appropriate predictive model. Age $\geq 50$ showed the highest increase in its share of total ill-defined causes from 1979 to 2002. The percentage of in-hospital deaths showed an inverse relationship with the percentage of deaths from ill-defined causes.

Cause of Death; Death Certificates; Mortality
Marli Ramos da Costa 1

Luiz Francisco Marcopito 2

\section{Introdução}

As estatísticas de mortalidade no Brasil padecem de pelo menos dois males: a subnotificação de óbitos - que atingiu 17,5\% no triênio 20002002 1, e a mortalidade por causas mal definidas, tema deste trabalho. São classificadas como de causas mal definidas as mortes súbitas de origem desconhecida, as mortes sem assistência, as mortes sem quaisquer outras especificações e as mortes relacionadas a afecções e sinais ou sintomas conhecidos como "mal definidos". Estes consistem em: "a) casos para os quais não se possa chegar a um diagnóstico mais preciso, mesmo depois que todos os fatos que digam respeito ao caso tenham sido investigados; $b$ ) sinais ou sintomas existentes no momento da primeira consulta que se mostrem de caráter transitório e cujas causas não possam ser determinadas; c) diagnósticos provisórios atribuídos a um paciente que não retorne à consulta para aprofundamento da investigação do diagnóstico ou para assistência; d) casos encaminhados a outros locais para investigação ou tratamento antes que o diagnóstico fosse feito; e) casos para os quais não foi possível estabelecer um diagnóstico mais preciso por qualquer outra razão; f) alguns sintomas para os quais se fornece informação complementar e que representam por si sós importantes problemas na assistência médica" 2 (p. 853). Os óbitos por causas mal definidas correspondem ao capítulo XVI da Classificação Internacional 
de Doenças, 9a revisão 3 (CID-9; Sintomas, sinais e afecções mal definidas) e ao capítulo XVIII da 10a revisão (CID-10) 2 (Sintomas, sinais e achados anormais de exames clínicos e de laboratório não classificados em outra parte).

A porcentagem de mortes por causas mal definidas (14\%) ocupou o segundo lugar no obituário nacional por grandes grupos de causas (capítulos da CID-10) no triênio 2000-2002, ficando atrás apenas dos óbitos por doenças do aparelho circulatório (27,4\%), e à frente das neoplasias (13\%) e das causas externas (12,7\%). Embora em declínio desde 1985 1,4 essa porcentagem no Brasil é das mais elevadas entre os países das Américas, abaixo apenas das de países como El Salvador, Paraguai e Haiti 5.

A considerável porcentagem de óbitos por causas mal definidas no Brasil faz com que a análise da mortalidade por causas definidas no país como um todo seja prejudicada. Há, porém, variações regionais que devem ser ressaltadas e, dentro de cada macro-região, diferenças marcantes entre Unidades da Federação (UF). Em 2002, por exemplo, essa porcentagem variou de $26,8 \%$ na Região Nordeste a 6,3\% na Região Sul, e de $40,5 \%$ no Estado da Paraíba a 3,2\% no Estado de Mato Grosso do Sul 1.

Quando essa porcentagem se concentra em determinadas faixas etárias, pode haver distorção dos indicadores de mortalidade por certas causas definidas de morte que são mais freqüentes em alguns grupos de idade. A identificação dessas faixas etárias, nesses casos, poderá ser de utilidade na apreciação das estatísticas de mortalidade por causas definidas de morte na população em estudo. Há indícios de que a mortalidade por causas mal definidas concentra-se em certos grupos de idade. Estudos focalizando as décadas de 1960 e 1970 mostraram que em alguns países da América Latina (Colômbia, Costa Rica, Chile, Guatemala, Paraguai) a porcentagem de óbitos por causas mal definidas foi maior nas primeiras e últimas faixas etárias 6,7,8 especialmente nos maiores de 65 anos e particularmente no grupo de 1-4 anos. Em 1983, no Chile, as doenças mal definidas estavam entre as três primeiras causas de morte nos idosos 9 .

Cornejo et al. 10 referem que, no Chile, as causas mal definidas se destacam especialmente nos maiores de 80 anos. Esses autores acreditam que a polipatologia dos idosos cria dificuldades para os médicos, que usam com freqüência os diagnósticos mal definidos. Relatam que a causa mal definida mais utilizada é a senilidade associada com outras enfermidades como aterosclerose generalizada, doença isquêmica crônica do coração e hipertensão arterial sistêmica. Segundo Vasconcelos 11, à medida que a idade aumenta, aumenta também a proporção de óbitos por causas mal definidas e isto se explica pela dificuldade de identificação da causa básica que iniciou o processo mórbido, visto o complexo encadeamento de causas em pessoas idosas. No entanto, comparando-se em 2002 a porcentagem de óbitos por causas mal definidas dentro de cada faixa etária a partir de 40 anos (40-49; 50-59; 60-69; 70-79 e $\geq 80$ anos) de duas UF brasileiras tão díspares como Paraíba e São Paulo, observa-se que a "regra do mais idoso" aplica-se bem à Paraíba (respectivamente: $33,4 \%$; 35,8\%; 41,3\%; 49,5\% e $61,6 \%$ ), mas nem tanto a São Paulo (respectivamente: 7,3\%; 7,4\%; 6,9\%; 6,6\%; e 8,3\%) 1 .

Chaimowicz 12 observou que em 1994, no Brasil, $60,4 \%$ de todas as declarações de óbito com causas mal definidas referiam-se a idosos (idade $\geq 60$ anos), cifra que aumentou para $67 \%$ em 2002 1. O problema com a mortalidade proporcional, porém, é que a soma de todas as parcelas tem que perfazer $100 \%$, não sendo possível dizer se o crescimento de uma se deve à redução de outra 13

Em vista do exposto, depreende-se que no Brasil a faixa etária mais afetada pelos óbitos por causas mal definidas ainda carece de identificação. Isso pode ter implicações importantes se, por exemplo, a idade $\geq 50$ anos (e não a $\geq 60$ anos) for a mais afetada, porque na faixa $\geq 50$ anos atualmente ocorrem mais de $2 / 3$ dos óbitos nacionais com idade conhecida, dos quais $39 \%$ devem-se a doenças classificadas nos capítulos IX (aparelho circulatório), II (neoplasias) e IV (endócrinas, nutricionais e metabólicas) da CID-10 14

Assim, considerando que as estatísticas nacionais de mortalidade de oito triênios (tempo) encontram-se disponíveis, e considerando as imensas disparidades regionais (espaço), há elementos para o estudo da mortalidade por causas mal definidas no tempo e espaço no Brasil, de 1979 a 2002, visando à avaliação das questões levantadas. Para isso, foram estabelecidos os seguintes objetivos: (a) descrever as modificações ocorridas nesses 24 anos; (b) identificar a faixa etária em que a porcentagem de óbitos por causas mal definidas mais se correlacionasse com a porcentagem total de mal definidas; (c) selecionar um modelo preditivo para a porcentagem de mal definidas na faixa etária assim identificada a partir da porcentagem total de mal definidas; (d) descrever as modificações observadas na contribuição percentual de cada faixa etária no total de óbitos por causas mal definidas e; (e) verificar se a porcentagem de óbitos ocorridas em hospital tem relação com a porcentagem de mortes por causas mal definidas. 


\section{Métodos}

Realizou-se estudo analítico com dados secundários (série histórica). A fonte de dados foi o Departamento de Informática do SUS (DATASUS; http://www.datasus.gov.br), que disponibiliza em meio eletrônico dados coletados das declarações de óbito de pessoas residentes no país. Foram estudados os óbitos por causas mal definidas de falecidos de 1979-2002, agrupados por grandes regiões brasileiras e por UF e, por faixa etária. Com relação ao Estado de Tocantins, criado em 1988, tais informações referem-se ao período 1989-2002.

Foram estudadas as seguintes variáveis dentro de cada UF de residência dos falecidos: (1) porcentagem anual de óbitos por causas mal definidas no total de óbitos registrados; (2) idade do óbito (em anos), agrupada em faixas; (3) porcentagem anual de óbitos por causas mal definidas dentro de cada faixa etária; (4) contribuição porcentual de faixas etárias selecionadas no total anual de óbitos por causas mal definidas, por ano-calendário e; (5) porcentagem de óbitos por todas as causas ocorridas em hospital.

$\mathrm{Na}$ análise estatística, primeiro foram construídos gráficos de dispersão entre "porcentagem anual de óbitos por causas mal definidas no total de óbitos registrados" e "porcentagem anual de óbitos por causas mal definidas dentro de cada faixa etária". Para esse fim a idade foi agrupada em 12 faixas: < 1 ; $1-4$; $5-9$; 10-14; 15-19; 20-29; 3039; 40-49; 50-59; 60-69; 70-79 e $\geq 80$.

Na exploração das faixas etárias, a análise estatística foi feita por correlação paramétrica e não-paramétrica. $\mathrm{O}$ uso de ambas as técnicas se justifica porque, enquanto a primeira assume normalidade na distribuição dos valores da variável, o que nem sempre é verdade, a segunda é independente da distribuição, já que emprega os postos ocupados pelos valores. Foram calculados os coeficientes de correlação de Pearson $(\mathrm{r})$, de Spearman $(\theta)$ e de Kendall $(\tau)$ - este último com correção para empates, utilizando-se a versão 10.0 do programa SPSS (SPSS Inc., Chicago, Estados Unidos) e técnica descrita em Rosner 15 e Siegel 16. A idéia foi verificar se as cinco faixas etárias clássicas (<1; 1-4; 5-19; 20-49 e $\geq 50$ anos) utilizadas por Moraes 17 poderiam ser apropriadas neste estudo.

A seguir a variável "porcentagem anual de óbitos por causas mal definidas no total de óbitos registrados" foi dividida em 11 categorias crescentes de $5 \%$ (exceto a mais elevada, que ficou aberta em $\geq 50 \%$ ). Em cada uma das cinco faixas etárias finalmente selecionadas foram descritos os valores mínimos e máximos de "porcentagem anual de óbitos por causas mal definidas dentro de cada faixa etária”, que foram dispostos graficamente, de modo a permitir a decisão sobre qual faixa etária, no conjunto, poderia ser a mais conveniente para a construção de um modelo preditivo.

Escolhida a faixa etária mais conveniente, foram testados os modelos preditivos de regressão linear $(\mathrm{y}=\mathrm{a}+\mathrm{bx})$ e cúbica $\left(\mathrm{y}=\mathrm{a}+\mathrm{b}_{1} \mathrm{x}+\mathrm{b}_{2} \mathrm{x}^{2}+\right.$ $\mathrm{b}_{3} \mathrm{x}^{3}$ ), a opção por um ou por outro feita com base tanto na impressão visual gráfica como nos valores de R2 (índice de adequação). A seguir foram determinadas as bandas que englobam $90 \%$ dos valores observados de "porcentagem anual de óbitos por causas mal definidas dentro da faixa etária" para cada valor observado de "porcentagem anual de óbitos por causas mal definidas no total de óbitos registrados". Nesta etapa foi outra vez utilizada a versão 10.0 do programa SPSS.

A contribuição percentual de idade ignorada no total de óbitos por causas mal definidas não se constituiu em empecilho para a realização deste estudo, pois no país a cifra foi de 1,3\% durante todo o período de estudo, variando de $0,6 \%$ na Região Sul a 2,2\% na Região Centro-Oeste 1.

\section{Resultados}

A Tabela 1 mostra que, no país como um todo, houve diminuição da porcentagem de óbitos por causas mal definidas a partir de 1985, saindo de cifras acima de $20 \%$ para algo em torno de $14 \%$ ao final do período de observação. Nas grandes regiões, houve significativa redução no Nordeste (de perto de $50 \%$ para menos de $30 \%$ ), Sul (de $15 \%$ para 6 a $7 \%$ ) e Centro-Oeste (de quase $20 \%$ para menos de $10 \%)$, mas pouca mudança no Norte (de $25 \%$ a $29 \%$ até 1995 , para $21 \%$ a $24 \%$ depois) e Sudeste (em torno de $9 \%$ durante todo o período).

Na Região Norte, destacam-se Roraima e Amapá, com porcentagens abaixo de $10 \%$ na maior parte do período estudado - se bem que o Amapá tenha piorado de 1997 em diante. No Tocantins houve significativa diminuição, de mais de $48 \%$ em 1989 para menos de 20\% nos últimos dois anos de observação. Nos demais estados da região, os índices oscilaram nas faixas de $11 \%$ a 24\% (Rondônia), de 16\% a 35\% (Amazonas) e de $25 \%$ a $32 \%$ (Pará).

Os estados da Região Nordeste foram os que apresentaram as maiores porcentagens de óbitos por causas mal definidas no total de óbitos registrados, a saber: Maranhão, 21\% a 58\%; Piauí, 23\% a $48 \%$; Ceará, $20 \%$ a $54 \%$; Rio Grande do Norte, $27 \%$ a $51 \%$; Paraíba, $40 \%$ a $68 \%$; Pernambuco, $19 \%$ a $55 \%$; Alagoas, $28 \%$ a $59 \%$; Sergipe, $29 \%$ a $55 \%$; e Bahia, $25 \%$ a $37 \%$. Dos 45 registros de por- 
Porcentagem anual de mortes por causas mal definidas no total de óbitos registrados, por região e Unidade da Federação (UF) de residência dos falecidos. Brasil, 1979-2002.

\begin{tabular}{|c|c|c|c|c|c|c|c|c|c|c|c|c|}
\hline \multirow{2}{*}{$\begin{array}{l}\text { Região e UF } \\
\text { de residência }\end{array}$} & \multicolumn{12}{|c|}{ Ano } \\
\hline & 1979 & 1980 & 1981 & 1982 & 1983 & 1984 & 1985 & 1986 & 1987 & 1988 & 1989 & 1990 \\
\hline Região Norte & 26,0 & 26,9 & 25,4 & 26,2 & 26,8 & 27,7 & 27,5 & 27,2 & 26,7 & 27,0 & 27,7 & 28,6 \\
\hline Rondônia & 22,9 & 24,2 & 20,0 & 17,3 & 14,3 & 13,6 & 12,4 & 11,9 & 12,1 & 11,1 & 11,9 & 14,0 \\
\hline Acre & 27,1 & 31,8 & 33,8 & 36,3 & 35,8 & 34,6 & 35,7 & 33,6 & 31,1 & 33,6 & 30,2 & 32,0 \\
\hline Amazonas & 17,7 & 16,0 & 15,7 & 21,0 & 22,8 & 29,5 & 29,6 & 30,1 & 27,0 & 30,9 & 32,9 & 35,4 \\
\hline Roraima & 5,5 & 8,9 & 8,9 & 10,8 & 14,9 & 16,1 & 14,8 & 14,5 & 16,4 & 10,4 & 7,8 & 8,9 \\
\hline Pará & 31,1 & 31,9 & 30,8 & 30,6 & 31,8 & 31,2 & 31,3 & 31,7 & 31,9 & 30,9 & 29,4 & 30,8 \\
\hline Amapá & 17,7 & 20,1 & 10,8 & 10,1 & 7,9 & 7,8 & 9,3 & 6,1 & 7,4 & 10,1 & 8,3 & 7,0 \\
\hline Tocantins & * & * & * & * & * & * & * & * & * & * & 48,5 & 41,9 \\
\hline Região Nordeste & 45,7 & 48,6 & 47,6 & 46,3 & 48,0 & 50,4 & 46,9 & 45,6 & 45,2 & 44,3 & 42,2 & 42,1 \\
\hline Maranhão & 20,6 & 22,2 & 22,4 & 46,6 & 54,7 & 57,6 & 53,7 & 52,2 & 50,3 & 50,6 & 47,9 & 49,5 \\
\hline Piauí & 22,9 & 28,4 & 34,5 & 41,9 & 44,4 & 46,5 & 47,7 & 48,2 & 48,4 & 44,1 & 40,4 & 39,5 \\
\hline Ceará & 38,8 & 40,4 & 41,3 & 41,1 & 51,4 & 53,6 & 48,7 & 46,3 & 47,2 & 45,4 & 45,0 & 44,4 \\
\hline Rio Grande do Norte & 29,5 & 51,4 & 51,1 & 40,9 & 40,3 & 47,6 & 46,7 & 41,8 & 43,0 & 40,7 & 37,8 & 37,3 \\
\hline Paraíba & 50,9 & 68,2 & 63,6 & 59,3 & 60,3 & 61,8 & 57,7 & 58,3 & 57,8 & 59,0 & 54,7 & 55,7 \\
\hline Pernambuco & 55,1 & 55,1 & 53,0 & 49,0 & 49,9 & 52,4 & 48,5 & 48,5 & 46,8 & 47,0 & 43,4 & 42,2 \\
\hline Alagoas & 59,2 & 58,3 & 55,2 & 54,1 & 53,1 & 55,5 & 51,3 & 49,8 & 49,0 & 47,3 & 46,5 & 47,1 \\
\hline Sergipe & 55,8 & 46,4 & 48,0 & 46,1 & 46,8 & 50,4 & 45,7 & 46,3 & 48,2 & 48,6 & 44,0 & 44,4 \\
\hline Bahia & 35,6 & 35,1 & 37,3 & 38,1 & 36,9 & 38,2 & 36,7 & 33,4 & 34,8 & 32,2 & 33,0 & 32,8 \\
\hline Região Sudeste & 10,0 & 9,6 & 9,1 & 8,8 & 8,9 & 8,8 & 9,5 & 9,0 & 8,6 & 8,4 & 8,3 & 8,7 \\
\hline Minas Gerais & 19,8 & 19,2 & 18,6 & 18,0 & 18,6 & 18,2 & 20,3 & 18,8 & 17,9 & 17,2 & 16,9 & 16,4 \\
\hline Espírito Santo & 28,6 & 25,3 & 20,5 & 18,5 & 19,5 & 19,2 & 21,6 & 22,1 & 21,7 & 21,4 & 21,6 & 20,8 \\
\hline Rio de Janeiro & 4,7 & 4,4 & 4,5 & 4,4 & 4,5 & 4,2 & 4,0 & 3,8 & 4,1 & 3,8 & 3,6 & 5,6 \\
\hline São Paulo & 5,9 & 6,0 & 5,6 & 5,6 & 5,6 & 5,8 & 6,0 & 6,0 & 5,7 & 5,9 & 6,1 & 6,2 \\
\hline Região Sul & 14,8 & 15,1 & 14,6 & 13,8 & 14,1 & 14,1 & 13,5 & 12,5 & 12,5 & 11,8 & 12,5 & 12,0 \\
\hline Paraná & 17,5 & 18,0 & 17,4 & 16,4 & 17,0 & 16,8 & 16,4 & 14,5 & 14,7 & 14,4 & 14,8 & 14,3 \\
\hline Santa Catarina & 21,1 & 20,9 & 21,0 & 19,5 & 19,4 & 19,9 & 18,5 & 18,1 & 18,4 & 16,0 & 16,0 & 17,3 \\
\hline Rio Grande do Sul & 9,9 & 10,2 & 9,6 & 9,4 & 9,5 & 9,6 & 9,0 & 8,7 & 8,4 & 8,1 & 9,2 & 8,0 \\
\hline Região Centro-Oeste & 16,7 & 16,3 & 18,5 & 19,4 & 18,8 & 18,2 & 18,6 & 17,7 & 17,8 & 17,0 & 14,3 & 12,4 \\
\hline Mato Grosso do Sul & 14,5 & 12,1 & 11,2 & 9,1 & 9,9 & 10,5 & 9,8 & 9,9 & 10,7 & 9,5 & 9,6 & 11,4 \\
\hline Mato Grosso & 26,2 & 24,5 & 25,5 & 24,0 & 20,9 & 21,2 & 18,5 & 17,1 & 16,0 & 17,0 & 16,8 & 16,3 \\
\hline Goiás & 20,7 & 21,5 & 24,9 & 26,6 & 26,1 & 24,8 & 26,5 & 25,0 & 25,3 & 24,1 & 19,9 & 15,9 \\
\hline Distrito Federal & 2,5 & 2,0 & 2,0 & 2,2 & 2,0 & 1,5 & 1,4 & 1,7 & 1,8 & 0,8 & 1,5 & 2,1 \\
\hline Brasil & 20,1 & 21,5 & 20,1 & 21,5 & 21,5 & 22,5 & 21,1 & 20,4 & 19,8 & 19,2 & 18,4 & 18,2 \\
\hline
\end{tabular}

* Até 1988 os óbitos do Tocantins foram contados em Goiás.

(continua)

centagens $\geq 50 \%$ de óbitos por causas mal definidas no período de estudo, todos ocorreram em UF da Região Nordeste, mas cifras tão elevadas quanto essas foram observadas pela última vez em 1999, na Paraíba.

Na Região Sudeste, chama atenção o Estado do Rio de Janeiro - que apresentava índices em torno de 4\% (de 1979 a 1989): a partir de 1990 a porcentagem foi se elevando, superando o patamar de 10\% de 1998 em diante. Houve redução gradual em Minas Gerais (de perto de $20 \%$ para menos de 14\%) e no Espírito Santo (de quase 30\% para menos de 20\%), e manutenção da porcentagem em cerca de 6\% no Estado de São Paulo durante todo o período de estudo.

Os três estados da Região Sul exibiram queda significativa ao longo do tempo de observação: no Paraná, de $18 \%$ para 5\%; em Santa Catarina, de $21 \%$ para $12 \%$; e, no Rio Grande do Sul, de $10 \%$ para $5 \%$. No Centro-Oeste, o Distrito Federal apresentou as menores porcentagens do país (por volta de $2 \%$ de 1979 a 1997, e cerca de $4 \%$ 


\begin{tabular}{|c|c|c|c|c|c|c|c|c|c|c|c|c|}
\hline \multirow{2}{*}{$\begin{array}{l}\text { Região e UF } \\
\text { de residência }\end{array}$} & \multicolumn{12}{|c|}{ Ano } \\
\hline & 1991 & 1992 & 1993 & 1994 & 1995 & 1996 & 1997 & 1998 & 1999 & 2000 & 2001 & 2002 \\
\hline Região Norte & 28,6 & 28,3 & 28,7 & 28,3 & 25,7 & 24,2 & 24,4 & 24,2 & 24,3 & 24,0 & 22,7 & 21,6 \\
\hline Rondônia & 16,6 & 17,9 & 17,0 & 16,7 & 18,4 & 18,3 & 15,7 & 14,7 & 15,4 & 12,9 & 12,5 & 11,1 \\
\hline Acre & 29,5 & 27,6 & 28,3 & 30,6 & 25,5 & 27,1 & 27,9 & 32,5 & 31,7 & 31,2 & 28,8 & 23,5 \\
\hline Amazonas & 31,4 & 31,0 & 33,2 & 30,8 & 25,6 & 23,1 & 24,6 & 22,8 & 23,8 & 25,2 & 24,8 & 25,2 \\
\hline Roraima & 8,3 & 7,4 & 6,8 & 7,6 & 8,3 & 4,6 & 7,8 & 3,9 & 9,0 & 9,1 & 6,2 & 4,4 \\
\hline Pará & 31,6 & 30,9 & 31,5 & 32,0 & 29,3 & 27,4 & 27,1 & 27,8 & 28,1 & 27,6 & 25,9 & 26,3 \\
\hline Amapá & 3,8 & 4,5 & 5,8 & 7,7 & 6,3 & 5,6 & 8,9 & 9,8 & 10,5 & 14,9 & 12,8 & 12,4 \\
\hline Tocantins & 41,4 & 37,4 & 34,0 & 32,7 & 32,4 & 30,3 & 30,2 & 28,6 & 24,6 & 21,2 & 19,8 & 11,8 \\
\hline Região Nordeste & 41,2 & 39,7 & 40,2 & 37,0 & 34,4 & 32,4 & 31,2 & 30,5 & 30,3 & 28,4 & 27,5 & 26,8 \\
\hline Maranhão & 49,4 & 49,6 & 49,6 & 47,4 & 42,4 & 40,1 & 39,6 & 40,0 & 40,7 & 36,1 & 39,5 & 40,3 \\
\hline Piauí & 40,8 & 38,3 & 36,0 & 37,4 & 36,0 & 34,1 & 32,3 & 32,7 & 31,4 & 30,7 & 29,6 & 25,9 \\
\hline Ceará & 42,5 & 41,9 & 43,5 & 43,5 & 38,1 & 30,9 & 28,9 & 24,0 & 21,9 & 20,2 & 21,5 & 25,5 \\
\hline Rio Grande do Norte & 36,3 & 37,4 & 38,5 & 35,8 & 30,3 & 29,9 & 29,7 & 29,5 & 29,5 & 27,6 & 28,0 & 27,0 \\
\hline Paraíba & 53,9 & 54,5 & 55,1 & 54,0 & 50,2 & 50,4 & 51,9 & 52,9 & 53,5 & 46,5 & 45,1 & 40,5 \\
\hline Pernambuco & 40,0 & 39,5 & 38,9 & 31,1 & 27,9 & 27,1 & 24,7 & 24,7 & 24,6 & 22,8 & 21,0 & 19,0 \\
\hline Alagoas & 43,9 & 45,2 & 43,7 & 42,4 & 40,0 & 41,2 & 38,2 & 34,8 & 34,4 & 29,1 & 28,0 & 28,2 \\
\hline Sergipe & 53,9 & 32,5 & 41,6 & 28,6 & 33,0 & 32,1 & 33,0 & 32,0 & 31,7 & 29,7 & 26,4 & 23,5 \\
\hline Bahia & 33,4 & 32,1 & 31,5 & 30,9 & 30,3 & 28,6 & 27,8 & 28,2 & 29,9 & 29,7 & 26,7 & 25,4 \\
\hline Região Sudeste & 9,3 & 9,7 & 9,8 & 9,8 & 9,7 & 9,2 & 9,1 & 10,0 & 10,1 & 9,8 & 9,6 & 9,1 \\
\hline Minas Gerais & 16,7 & 16,8 & 16,3 & 15,5 & 15,0 & 14,4 & 14,2 & 14,8 & 16,1 & 14,2 & 13,7 & 13,1 \\
\hline Espírito Santo & 21,7 & 22,1 & 19,9 & 19,8 & 20,2 & 19,9 & 18,5 & 19,4 & 19,3 & 17,6 & 16,4 & 11,8 \\
\hline Rio de Janeiro & 7,8 & 8,4 & 9,8 & 9,9 & 9,8 & 9,6 & 9,8 & 11,0 & 10,9 & 11,5 & 11,2 & 10,8 \\
\hline São Paulo & 5,8 & 6,1 & 6,1 & 6,5 & 6,6 & 6,0 & 6,1 & 6,7 & 6,6 & 6,6 & 6,7 & 6,5 \\
\hline Região Sul & 10,6 & 9,8 & 9,8 & 9,5 & 9,4 & 8,9 & 7,7 & 7,7 & 7,1 & 6,3 & 6,3 & 6,3 \\
\hline Paraná & 12,3 & 11,2 & 11,0 & 10,7 & 10,8 & 9,8 & 7,3 & 7,1 & 6,6 & 5,4 & 5,1 & 4,6 \\
\hline Santa Catarina & 16,0 & 14,3 & 14,5 & 14,7 & 15,1 & 15,3 & 13,7 & 14,2 & 13,0 & 12,6 & 12,0 & 11,3 \\
\hline Rio Grande do Sul & 7,1 & 7,0 & 6,9 & 6,5 & 6,1 & 5,6 & 5,4 & 5,6 & 5,0 & 4,4 & 4,9 & 5,7 \\
\hline Região Centro-Oeste & 12,6 & 13,1 & 12,9 & 12,8 & 13,3 & 10,8 & 10,5 & 10,6 & 10,1 & 8,5 & 7,9 & 6,6 \\
\hline Mato Grosso do Sul & 10,3 & 10,7 & 11,2 & 10,4 & 10,4 & 10,9 & 10,3 & 10,1 & 9,2 & 8,5 & 5,5 & 3,2 \\
\hline Mato Grosso & 18,9 & 19,6 & 20,0 & 19,0 & 15,2 & 10,0 & 8,7 & 9,2 & 7,5 & 7,9 & 8,9 & 8,2 \\
\hline Goiás & 15,3 & 15,6 & 15,2 & 16,0 & 18,0 & 14,7 & 14,3 & 13,9 & 13,6 & 10,2 & 9,5 & 8,0 \\
\hline Distrito Federal & 1,9 & 2,3 & 1,4 & 1,6 & 1,5 & 1,2 & 2,0 & 3,9 & 4,9 & 4,7 & 5,1 & 4,6 \\
\hline Brasil & 18,2 & 17,8 & 18,0 & 17,0 & 16,2 & 15,1 & 14,7 & 15,1 & 15,1 & 14,3 & 14,1 & 13,7 \\
\hline
\end{tabular}

* Até 1988 os óbitos do Tocantins foram contados em Goiás.

a 5\% depois). No Mato Grosso do Sul, as cifras baixaram de $14 \%$ a $12 \%$ (em 1979-1982) para $9 \%$ a 11\% (em 1983-1999), com queda mais expressiva a partir de 2000. A diminuição também foi significativa em Mato Grosso (de 26\% para menos de 10\%) e em Goiás a partir de 1989 (de 20\% para menos de $10 \%$ ).

A Tabela 2 apresenta os coeficientes de correlação [não paramétrica $(\tau, \theta)$ e paramétrica $(r)$ ] em 21 faixas etárias. Observa-se que as duas faixas etárias mais jovens ( $<1$ e de 1-4 anos) apre$\operatorname{sentam} \tau \leq 0,75$. Das seis faixas etárias construídas entre 5 e 19 anos de idade, a faixa de 5 a 19 é a que exibe a maior correlação $(\tau=0,75)$. Das seis faixas etárias construídas entre 20-49 anos, a faixa de 20-49 tem $\tau$ mais elevado (0,83 - em- patando com outras duas). Finalmente, das sete faixas etárias construídas entre os mais idosos, a faixa $\geq 50$ anos é a que mostrou a mais elevada correlação $(\tau=0,93)$. Em vista desses resultados, as faixas etárias selecionadas, além daquelas de $<1$ ano e de 1-4 anos, foram a de 5-19, a de 20-49 e a de $\geq 50$ anos.

A Figura 1 mostra os gráficos de dispersão naquelas cinco faixas etárias selecionadas (todos os gráficos estão na mesma escala aritmética). Na Figura 2 observa-se que a faixa etária $\geq 50$ anos é a mais passível de modelagem estatística por apresentar, no conjunto, menor amplitude de variação em qualquer categoria de "porcentagem total de óbitos por causas mal definidas". 
Tabela 2

Coeficientes de correlação de Kendall $(\tau)$, de Spearman $(\theta)$ e de Pearson $(r)$ entre "porcentagem total de causal mal definidas" e "porcentagem de causas mal definidas dentro da faixa etária", em todas as unidades da federação, em 21 faixas etárias. Brasil, 1979-2002.

\begin{tabular}{|c|c|c|c|}
\hline \multirow{2}{*}{$\begin{array}{l}\text { Faixa etária } \\
\text { (em anos) }\end{array}$} & \multicolumn{3}{|c|}{ Coeficientes de correlação } \\
\hline & $\tau$ & $\theta$ & $r$ \\
\hline$<1$ & 0,72 & 0,88 & 0,85 \\
\hline $1-4$ & 0,75 & 0,91 & 0,89 \\
\hline $5-9$ & 0,70 & 0,88 & 0,87 \\
\hline $5-14$ & 0,72 & 0,89 & 0,88 \\
\hline 5-19 & 0,75 & 0,91 & 0,90 \\
\hline $10-14$ & 0,68 & 0,85 & 0,84 \\
\hline $10-19$ & 0,74 & 0,91 & 0,89 \\
\hline $15-19$ & 0,71 & 0,89 & 0,88 \\
\hline $20-29$ & 0,76 & 0,92 & 0,91 \\
\hline $20-39$ & 0,80 & 0,95 & 0,92 \\
\hline $20-49$ & 0,83 & 0,96 & 0,94 \\
\hline $30-39$ & 0,80 & 0,95 & 0,92 \\
\hline $30-49$ & 0,83 & 0,96 & 0,93 \\
\hline $40-49$ & 0,83 & 0,96 & 0,93 \\
\hline $50-59$ & 0,86 & 0,97 & 0,95 \\
\hline$\geq 50$ & 0,93 & 0,99 & 0,99 \\
\hline $60-69$ & 0,89 & 0,98 & 0,97 \\
\hline$\geq 60$ & 0,92 & 0,99 & 0,98 \\
\hline $70-79$ & 0,91 & 0,99 & 0,98 \\
\hline$\geq 70$ & 0,91 & 0,99 & 0,98 \\
\hline$\geq 80$ & 0,89 & 0,98 & 0,97 \\
\hline
\end{tabular}

A Figura 3 mostra as bandas teóricas de confiança de $90 \%$ (área em cinza), a partir dos modelos de regressão linear e cúbica. Nota-se que, de fato - como sugerem os valores de $\mathrm{R}^{2}$, de 0,973 para o modelo linear e de 0,980 para o cúbico, o modelo cúbico adapta-se melhor do que o linear aos valores observados. Por regressão linear, as bandas de $90 \%$, inferior e superior, respectivamente, são obtidas das equações:

$\mathrm{y}=-3,507415+1,130507 x$

$\mathrm{y}=6,138925+1,130759 \mathrm{x}$

E, por regressão cúbica, as bandas de $90 \%$, inferior e superior, respectivamente, são obtidas das equações:

$\mathrm{y}=-5,410502+1,341183 \mathrm{x}-0,000534 \mathrm{x}^{2}-$ $0,0000634167909 x^{3}$

$\mathrm{y}=2,940863+1,344927 \mathrm{x}-0,000796 \mathrm{x}^{2}-$ $0,0000593253212 x^{3}$

Por regressão linear, a previsão da média é obtida pela equação:

$\mathrm{y}=1,315755+1,130633 \mathrm{x}$

E, por regressão cúbica, a previsão da média é obtida pela equação:

$y=-1,234820+1,343055 x-0,000685 x^{2}-$ $0,0000613710560 x^{3}$
Assim, se, por exemplo, a porcentagem total de óbitos por causas mal definidas (x) for $30 \%$, $90 \%$ dos valores de porcentagem de óbitos por causas mal definidas na idade $\geq 50$ anos (y) estarão entre $30,4 \%$ e $40,1 \%$ (pelo modelo linear) ou entre $32,6 \%$ e $42,4 \%$ (pelo modelo cúbico). A estimativa pontual de y será de $35,2 \%$ (pela regressão linear) e de 36,8\% (pela regressão cúbica).

Entre as cinco faixas etárias selecionadas para análise (< 1 ano, 1-4 anos, 5-19 anos, 20-49 anos e $\geq 50$ anos), a que apresentou a mais crescente contribuição percentual no total de causas mal definidas no país foi a de $\geq 50$ anos (de $41,3 \%$, em 1979, para $84,3 \%$, em 2002), contrapondo-se com as faixas etárias de $<1$ ano (de 30,9\% em 1979 para $2,4 \%$ em 2002) e de $1-4$ anos (de $14,4 \%$ para $1,4 \%$ em 2002).

Outro resultado de interesse obtido neste estudo refere-se à porcentagem anual de mortes por todas as causas ocorridas em hospital, que exibiu relação inversa com a cifra de óbitos por causas mal definidas. No período 1979-1987 (quando a porcentagem nacional de mal definidas oscilou entre $19,8 \%$ e $22,5 \%$ ), a porcentagem de mortes em ambiente hospitalar variou entre $57 \%$ e 58,3\%. A partir de 1988 (quando as mal definidas decresceram paulatinamente de $19,2 \%$ para $13,7 \%$ ), a porcentagem de óbitos em hospital aumentou gradativamente de 59,3\% para $67,3 \%$.

\section{Discussão}

A mortalidade proporcional por causas mal definidas dentro do total de óbitos informados é um dos mais utilizados indicadores da qualidade das informações sobre mortalidade de uma região geográfica. Embora possa ser artificialmente afetada pelo efeito proporcional dos óbitos por causas definidas (a soma de todas as porcentagens de mortalidade proporcional deve perfazer $100 \%)$, é preferida à respectiva taxa quando a cobertura de óbitos informados é baixa.

Neste estudo foi avaliada uma série histórica de 24 anos de todas as UF do Brasil, abrangendo variações no tempo e no espaço que podem representar diferentes estágios de desenvolvimento sócio-econômico de várias partes do mundo. De fato, foram observadas porcentagens tão elevadas quanto 68,2\% em 1980 na Paraíba - maiores do que as de alguns países africanos onde há estatísticas disponíveis -, como tão baixas quanto $0,8 \%$ em 1988 no Distrito Federal - menores do que as de alguns países da Europa setentrional 18 e da América anglofônica e de Cuba 5 .

Apesar da melhora observada no país como um todo ao longo de 1979-2002, as regiões Norte 
CAUSAS MAL DEFINIDAS DE MORTALIDADE

1007

Figura 1

Valores observados de "porcentagem total de óbitos por causas mal definidas" (eixo x) e "porcentagem de óbitos por causas mal definidas na faixa etária" (eixo y), em cinco faixas etárias selecionadas, todas as Unidades da Federação (UF), por ano. Brasil, 1979-2002.

1a) $<1$ ano

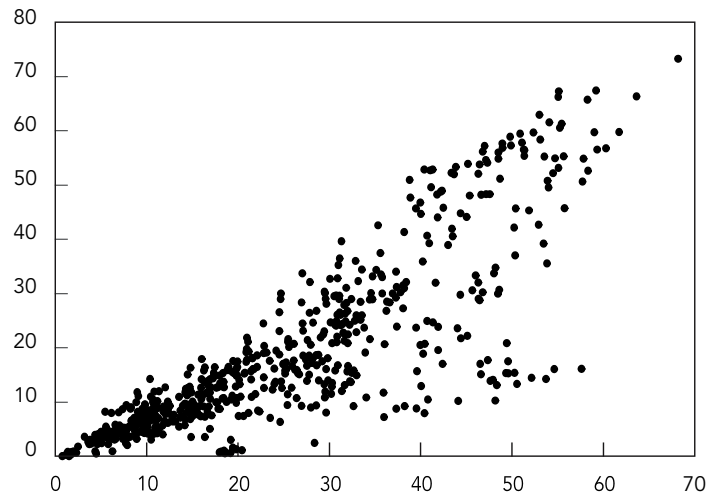

1c) $5-19$ anos

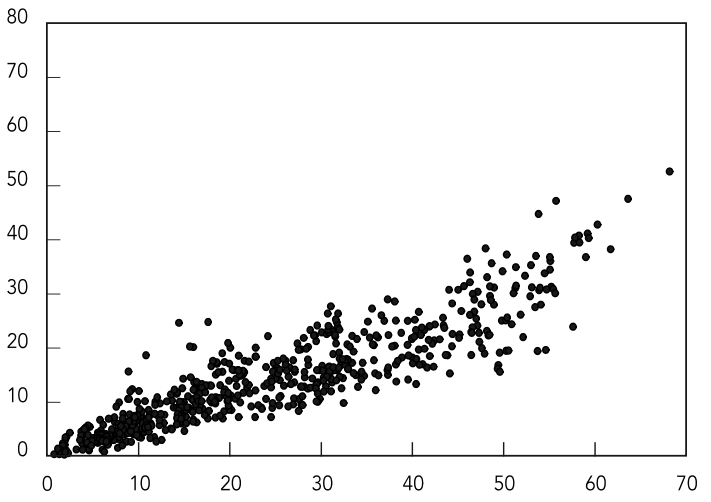

1e) $\geq 50$ anos

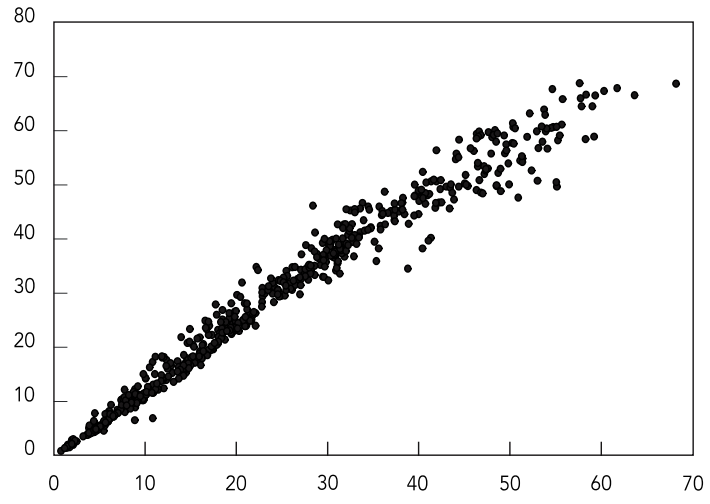

1b) 1-4 anos

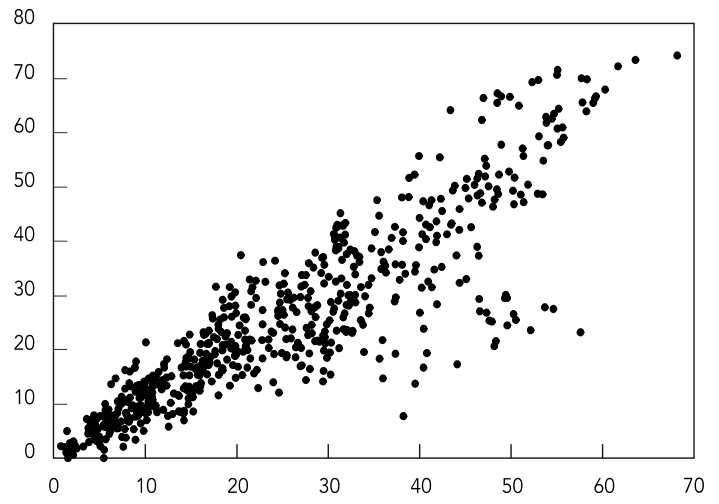

1d) 20-49 anos

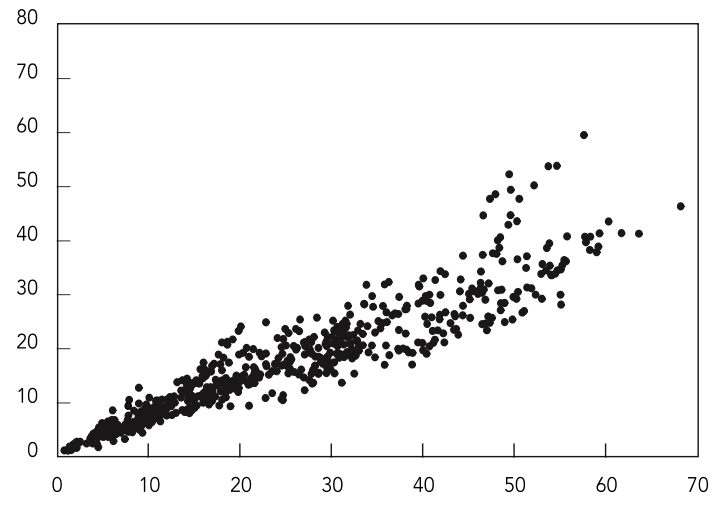

Cad. Saúde Pública, Rio de Janeiro, 24(5):1001-1012, mai, 2008 
Figura 2

Valores mínimos e máximos da "porcentagem de óbitos por causas mal definidas" (eixo y) em cada categoria de "porcentagem total de óbitos por causas mal definidas" (eixo x), por cinco faixas etárias selecionadas, todas as Unidades da Federação (UF), por ano. Brasil, 1979-2002.

2a) $<1$ ano

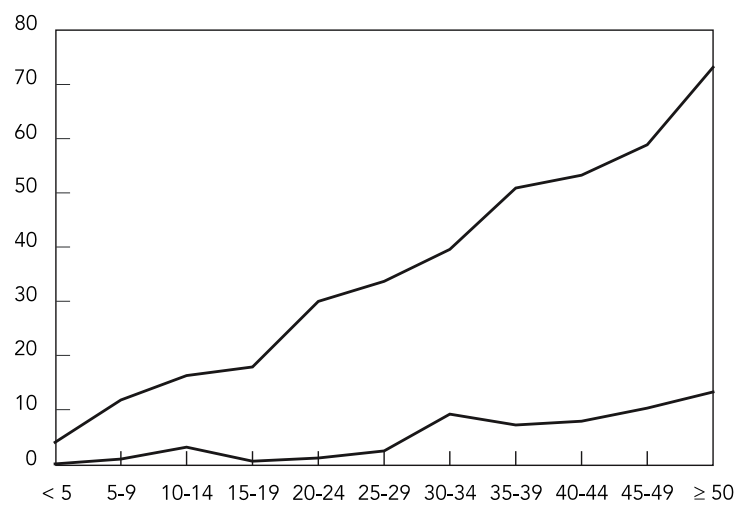

2c) 5-19 anos

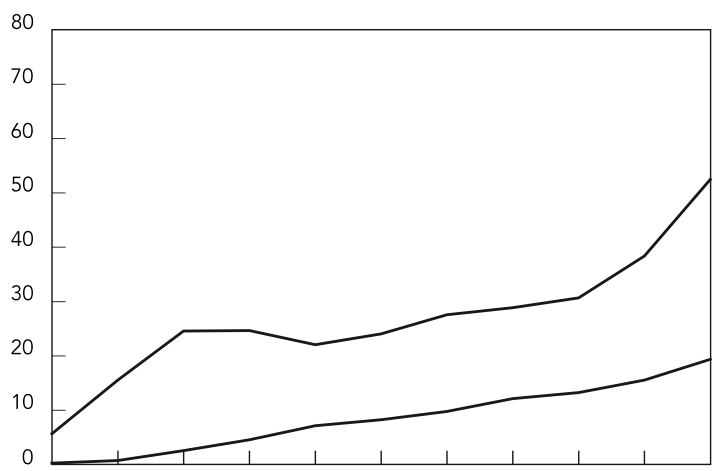

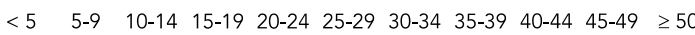

2b) 1-4 anos

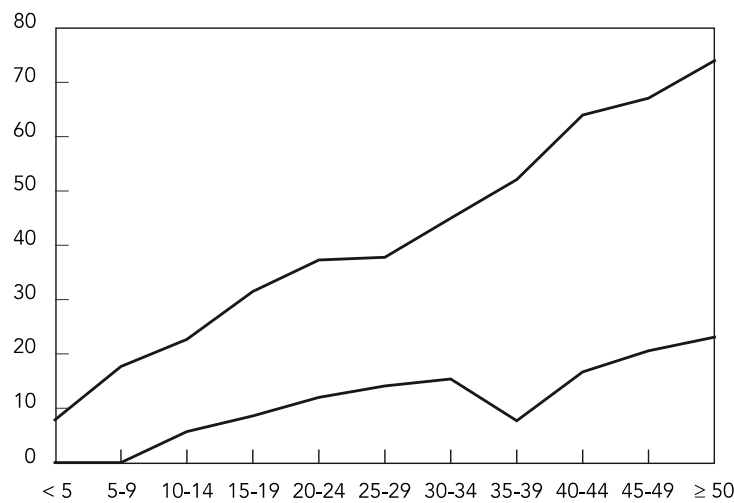

2d) 20-49 anos

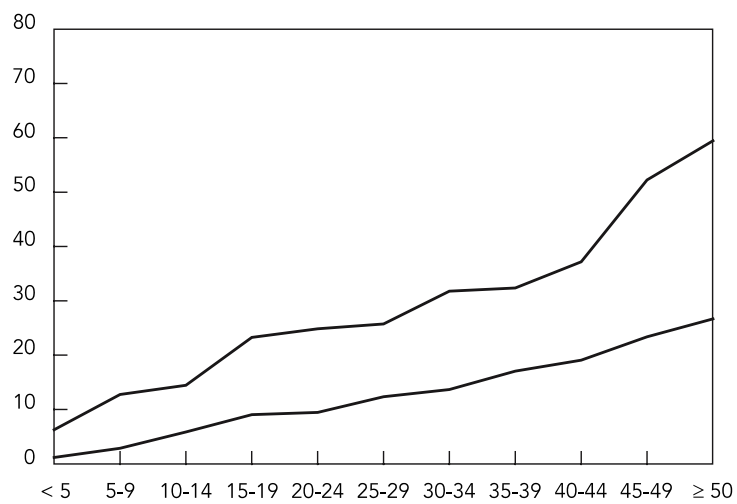

2e) $\geq 50$ anos

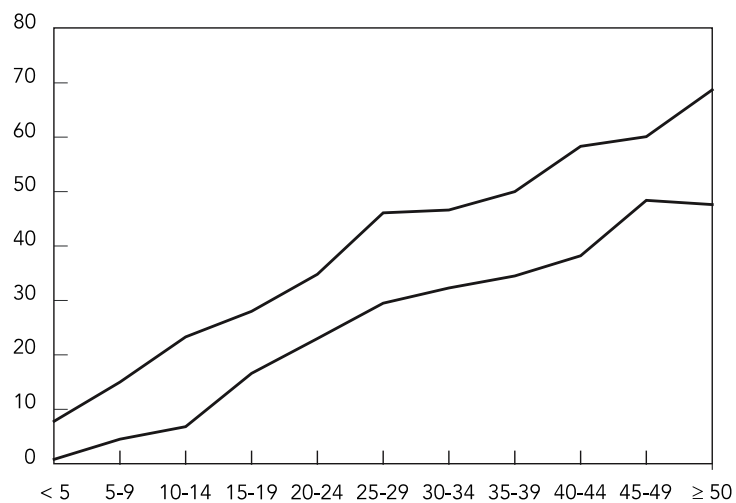


Bandas teóricas de confiança de 90\% (áreas em cinza), a partir dos modelos de regressão linear (3a) e cúbica (3b). Os pontos são os valores observados, todas as Unidades da Federação (UF). Brasil, 1979-2002.

3a) regressão linear

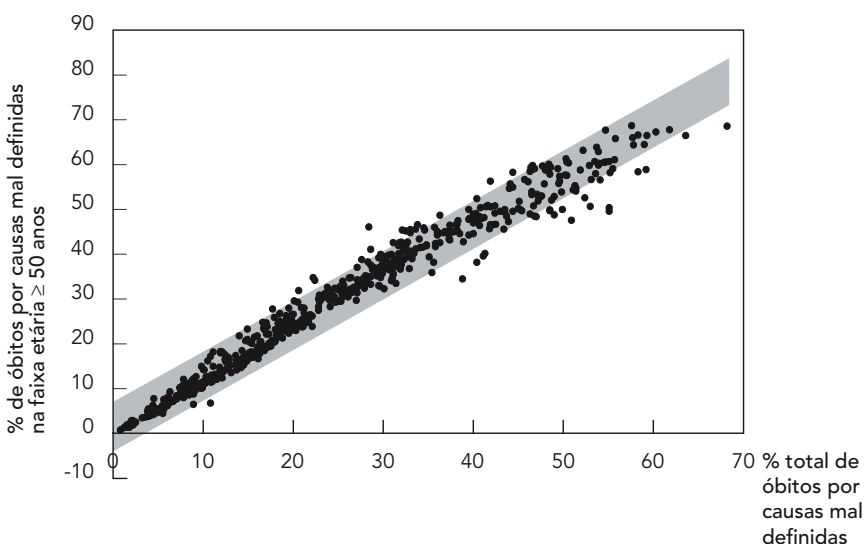

3b) regressão linear

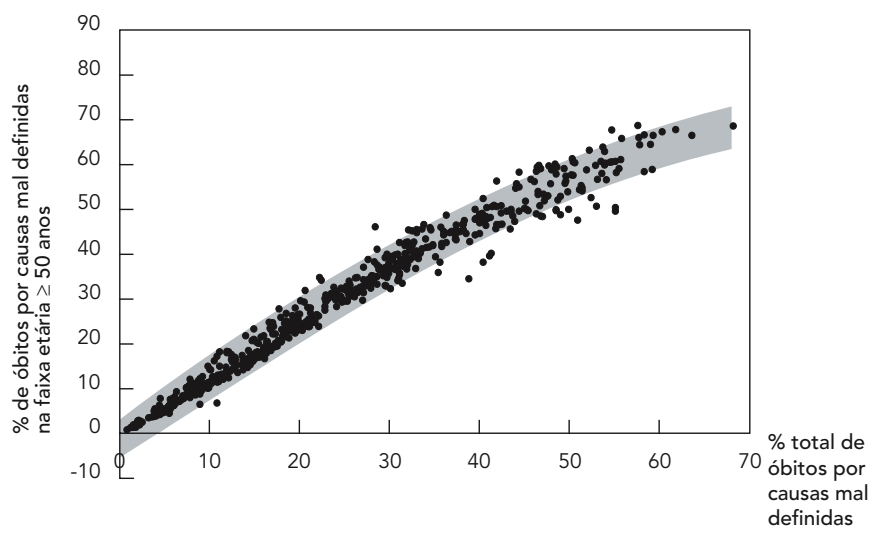

e Nordeste terminaram o período de observação com cifras acima de $20 \%$ - se bem que isso possa ser comemorado para o Nordeste, que partiu de porcentagens próximas a $50 \%$. O Sudeste, que se manteve com $9 \%$ a $10 \%$, acabou sendo superado pelo Sul e pelo Centro-Oeste, que atingiram o patamar de $6 \%$ a $7 \%$ nos últimos anos do estudo.

Dentro desse cenário geral de queda na porcentagem de óbitos por causas mal definidas destoa o Rio de Janeiro, que voltou a apresentar cifras crescentes a partir de 1990, elevação que mostra relação temporal com a publicação da Resolução SES/RJ no. 550, de 1990 19,20. Essa resolução estabelece que cabe ao médico plantonista ou substituto atestar o óbito de todo paciente que venha a falecer por causa natural a caminho ou nas dependências do pronto-socorro ou ambulatório público ou privado, e que não havendo suspeita de óbito por causa violenta, deverá ser declarada na parte I do atestado médico "causa indeterminada”. Já o caso do Distrito Federal, que a partir de 1998 apresentou cifras de mal definidas até então não registradas, merece um estudo especial para averiguar as razões daquela mudança - consideração que também se aplica ao Amapá, de 1999 em diante.

O estudo da correlação entre a mortalidade proporcional por faixa etária e o total de mal definidas revelou que, como era de se esperar, a mortalidade proporcional por faixa etária apre- senta tendência positiva de crescimento conforme aumenta o total da mortalidade proporcional por causas mal definidas (as partes correlacionadas com o todo). A julgar pelos gráficos de dispersão, o coeficiente $\tau$ de Kendall - provavelmente por levar em conta os empates, gera medidas mais fidedignas de correlação. Exemplo típico é o da idade < 1 ano: graficamente há pouca correlação entre a porcentagem total de mal definidas, quando esta está entre $30 \%$ e $60 \%$, e a porcentagem de mal definidas nessa faixa etária, mas os coeficientes de Spearman $(\theta)$ e de Pearson (r) para todo o intervalo são muito elevados (0,88 e 0,85 , respectivamente), enquanto o coeficiente $\tau$ de Kendall é mais conservador $(0,72)$. Fenômeno semelhante ocorreu com a idade 1-4 anos.

Com referência às quatro faixas etárias mais idosas (50-59, 60-69, 70-79 e $\geq 80$ anos), o estudo de correlação com a porcentagem total de mal definidas mostrou que essas podem ser agrupadas numa única faixa aberta de $\geq 50$ anos. Isso tem conseqüência bastante positiva porque essa faixa etária aberta reúne maior número de óbitos do que aquelas isoladamente, e pode servir como indicador tanto para áreas muito como pouco desenvolvidas. O estudo de correlação também evidenciou que as faixas etárias de 20 29, 30-39 e 40-49 anos, bem como as de 5-9, 1014 e 15-19 anos, podem ser agrupadas - respec- 
tivamente, nas categorias de 20-49 e 5-19 anos para o estudo da mortalidade proporcional por causas mal definidas. Essa evidência permitiu que as clássicas faixas etárias de mortalidade (<1, 1-4, 5-19, 20-49 e $\geq 50$ anos) de Moraes 17, tão conhecidas pelos epidemiologistas e demógrafos brasileiros, pudessem também ser utilizadas no presente estudo.

Tanto a análise estatística por correlação como a análise visual por gráficos (de dispersão e de valores mínimos e máximos por categorias de porcentagens do total de mal definidas) mostraram que a porcentagem de óbitos por causas mal definidas na faixa etária $\geq 50$ anos é a que mostra a maior correlação com a porcentagem total de óbitos por causas mal definidas. Utilizando técnicas de regressão foi possível selecionar um modelo que pode predizer, com $90 \%$ de certeza, a porcentagem de óbitos por causas mal definidas na faixa etária $\geq 50$ anos de acordo com a porcentagem total de óbitos por causas mal definidas. Acredita-se que este seja o resultado mais importante obtido neste estudo, pois na idade $\geq$ 50 anos é que se concentra a maior mortalidade por doenças crônicas não transmissíveis 14 .

Embora a regressão cúbica seja a que forneça as mais precisas previsões para a porcentagem de causas mal definidas na idade $\geq 50$ anos, este modelo matemático é um tanto complexo para o uso rotineiro. Já a regressão linear (bem mais simples do que a cúbica) só não pode dar muito boas previsões quando a porcentagem total de óbitos por causas mal definidas está acima de $50 \%$, situação que pela última vez ocorreu no Brasil em 1999 (53,5\%), na Paraíba.

A contribuição porcentual da faixa etária $\geq 50$ anos no total de óbitos por causas mal definidas aumentou em todas as grandes regiões do país, situação que pode ser explicada pela acentuada diminuição na porcentagem de óbitos por causas mal definidas nas faixas etárias < 1 ano e 1-4 anos (o efeito proporcional, já citado).

A partir de informações retiradas do DATASUS 1, depreende-se que a redução na porcentagem de óbitos por causas mal definidas observada no Brasil, de 20,1\% em 1979 para $13,7 \%$ em 2002, pode ser explicada apenas em parte pelo aumento na porcentagem de óbitos por causas externas (de 9,2\% em 1979 para $12,9 \%$ em 2002, com pico de $13,2 \%$ em 1997). Além disso, conforme observado neste estudo, a porcentagem de mortes ocorridas em hospital guardou relação inversa com a mortalidade proporcional por causas mal definidas, e a mu- dança na CID - implementada em 1996 para os dados de mortalidade, não alterou a tendência nacional de queda na porcentagem de mal definidas - que já vinha ocorrendo desde meados da década de 1980. Esse conjunto de informações leva a crer que a maior parte do progresso alcançado deve mesmo ter acontecido por aumento na determinação das causas naturais de morte.

É muito provável que as porcentagens de óbitos por causas mal definidas nas UF com as piores cifras diminuam à medida que aumente $o$ acesso à assistência médica na situação de risco de morte, particularmente em ambiente hospitalar - onde há, em teoria, mais recursos diagnósticos. O lado ruim é que a maioria dos óbitos por causas mal definidas ocorridos em hospitais não terá suas causas desvendadas mesmo após estudos especiais, como ocorreu no trabalho recente de Teixeira et al. 20 no Rio de Janeiro. Aliás, como já mencionado, o Estado do Rio de Janeiro tem o viés da Resolução SES/RJ no. 550, de 1990, ainda em vigor 19,20.

Concluindo, embora o Brasil ainda apresente elevada porcentagem de mortes por causas mal definidas entre os óbitos registrados, verificase que o país tem melhorado essa estatística ao longo dos anos. Ainda há focos de resistência, como alguns estados das regiões Nordeste e Norte, nessa ordem, mas avanços significativos são observados em Minas Gerais, Espírito Santo e regiões Sul e Centro-Oeste. Além disso, em anos recentes, porcentagens melhores do que as aceitáveis $(<10 \%)$ são observadas em Roraima, São Paulo, Paraná, Rio Grande do Sul, e todas as UF da Região Centro-Oeste. É preciso lembrar, no entanto, que a mais recente estimativa 21 da subnotificação de óbitos em 2002 ainda era elevada para algumas dessas UF, como Roraima (35,7\%), Mato Grosso $(9,8 \%)$ e Goiás $(13,5 \%)$ - além das altas cifras para as regiões Norte (28\%) e Nordeste $(29,8 \%)$.

A constatação de que a porcentagem de óbitos por causas mal definidas na faixa etária $\geq 50$ anos pode ser prevista pela porcentagem total de óbitos por causas mal definidas - qualquer que seja o valor desta última, é promissora, porque teoricamente pode ser utilizada para qualquer região geográfica considerada - tanto desenvolvida, como em desenvolvimento ou subdesenvolvida. Resta saber se esse modelo preditivo pode valer para cada um dos sexos isoladamente, já que certas causas de morte (como as externas, por exemplo) afetam mais os homens do que as mulheres. 


\section{Resumo}

Estudou-se a porcentagem de mortes por causas mal definidas no Brasil, de 1979 a 2002, com os objetivos de: (a) descrever modificações nesses 24 anos; (b) identificar a faixa etária em que a porcentagem de óbitos por mal definidas mais se correlaciona com a porcentagem total de mal definidas; (c) selecionar um modelo preditivo para a porcentagem de mal definidas nessa faixa etária, a partir da porcentagem total de mal definidas; (d) descrever modificações na contribuição de cada faixa etária no total de mal definidas; (e) verificar se a porcentagem de óbitos ocorridos em hospital relaciona-se com a porcentagem de mortes por mal definidas. Observou-se que a porcentagem total de óbitos por causas mal definidas diminuiu no Brasil. A porcentagem de mal definidas na faixa etária $\geq 50$ anos é a que mais se correlaciona com o total de mal definidas: a regressão cúbica é o modelo mais adequado para predição. Essa faixa etária foi a que mais aumentou sua contribuição no total de mal definidas, e a porcentagem de óbitos ocorridos em hospital guardou relação inversa com a porcentagem de mal definidas.

Causa de Morte; Atestado de Óbito; Mortalidade

\section{Referências}

1. Departamento de Informática do SUS. Informações de saúde: mortalidade. http://tabnet. datasus.gov.br/cgi/deftohtm.exe?sim/cnv/obtuf. def (acessado em 26/Abr/2005).

2. Organização Mundial da Saúde. Classificação estatística internacional de doenças e problemas relacionados à saúde, 10a revisão. v. 1. São Paulo: Centro Colaborador da OMS para a Classificação de Doenças em Português; 1995.

3. Organização Mundial da Saúde. Manual da classificação estatística internacional de doenças, lesões e causas de óbitos - nona conferência de revisão. São Paulo: Centro Brasileiro de Classificação de Doenças em Português; 1985.

4. Paes NA. A mortalidade por causas no Brasil: qualidade e comportamento dos dados. In: Anais do X Encontro Nacional de Estudos Populacionais. Belo Horizonte: Associação Brasileira de Estudos Populacionais; 1996. p. 1945-69.

5. Organización Panamericana de la Salud. Sobre la estimación de tasas de mortalidad para países de la región de las Américas. Bol Epidemiol (Wash) 2003; 24:1-5.

6. Chackiel J. La investigación sobre causas de muerte en la América Latina. Notas Poblac 1987; (44): 9-30.

\section{Colaboradores}

M. R. Costa fez o levantamento bibliográfico, a coleta de dados e a redação do artigo. L. F. Marcopito realizou a análise estatística e fez revisão final do artigo.
7. Jaspers-Faijer D, Orellana H. Evaluación del uso de las estadisticas vitales para estudios de causas de muerte en América Latina. Notas Poblac 1994; (60):47-77.

8. Aidar T. Registro de óbitos com causa mal definida: atenção especial para o caso da população feminina. Brasil 1979 a 1995. In: Anais do XII Encontro de Estudos Populacionais [CD-ROM]. Caxambu: Associação Brasileira de Estudos Populacionais; 2000.

9. Kirschbaum A, Arias EC. Mortalidad del senescente en Chile. Cuad Méd-Soc (Santiago de Chile) 1988; 29:134-8.

10. Cornejo EA, Kirschbaum AK, Castillo Paola C. Causas de muerte mal definidas en senescentes. Rev Méd Chile 1990; 118:92-8.

11. Vasconcelos AMN. Estatísticas de mortalidade por causas: uma avaliação da qualidade da informação In: Anais do X Encontro Nacional de Estudos Populacionais. Belo Horizonte: Associação Brasileira de Estudos Populacionais; 1996. p. 151-65.

12. Chaimowicz F. A saúde dos idosos brasileiros às vésperas do século XXI: problemas, projeções e alternativas. Rev Saúde Pública 1997; 31:184-200. 
13. Marcopito LF, Berlin JA. A method for calculating age-weighted death proportions for comparison purposes. Int J Epidemiol 1998; 27:1044-52.

14. Secretaria de Vigilância em Saúde, Ministério da Saúde. Saúde Brasil 2005: uma análise da situação de saúde. Brasília: Ministério da Saúde; 2005.

15. Rosner B. Fundamentals of biostatistics. $4^{\text {th }}$ Ed. Belmont: Duxbury Press; 1995.

16. Siegel S. Estatística não paramétrica para as ciências do comportamento. São Paulo: McGraw-Hill do Brasil; 1975.

17. Moraes N. Níveis de saúde de coletividades brasileiras. Revista do Serviço Especial de Saúde Pública $1959 ; 10: 403-97$.

18. Mathers CD, Fat DM, Inoue M, Rao C, Lopez AD. Counting the dead and what they died from: an assessment of the global status of cause of death data. Bull World Health Organ 2005; 83:171-7.
19. Reis ACGV. Mortalidade por causas mal definidas na Região Metropolitana do Rio de Janeiro, de 1980 a 1995 [Dissertação de Mestrado]. Rio de Janeiro: Escola Nacional de Saúde Pública, Fundação Oswaldo Cruz; 1999.

20. Teixeira CLS, Klein CH, Bloch KV, Coeli CM. Reclassificação dos grupos de causas prováveis dos óbitos de causa mal definida, com base nas Autorizações de Internação Hospitalar no Sistema Único de Saúde, Estado do Rio de Janeiro, Brasil. Cad Saúde Pública 2006; 22:1315-24.

21. Departamento de Informática do SUS. Informações de saúde: IDB-2006. http://tabnet.datasus. gov.br/cgi/idb2006/matriz.htm (acessado em 29/ Mai/2007).

Recebido em 10/Nov/2006

Versão final reapresentada em 12/Jun/2007 Aprovado em 10/Out/2007 